\title{
What is the role of post acute EEG in prediction of late neurological outcome in severe disorders of consciousness?
}

\author{
Maenia Scarpino ${ }^{1,2}$, Francesco Lolli³, Giovanni Lanzo ${ }^{2}$ \& Antonello Grippo*,1,2 \\ ${ }^{1}$ IRCCS Fondazione Don Carlo Gnocchi, Firenze \\ ${ }^{2}$ SODC Neurofisiopatologia, Dipartimento Neuromuscolo-Scheletrico e degli Organi di Senso, AOU Careggi, Firenze \\ ${ }^{3}$ Dipartimento di Scienze Biomediche Sperimentali e Cliniche Mario Serio, Università degli studi di Firenze \\ *Author for correspondence: agrippo@unifi.it
}

First draft submitted: 9 September 2019; Accepted for publication: 20 September 2019; Published online: 14 January 2020

Keywords: acute severe injuries $\bullet$ brain $\bullet$ disorders of consciousness $\bullet$ electroencephalography (EEG) • emergence from minimally conscious state $\bullet$ intensive rehabilitation unit $\bullet$ minimally conscious state $\bullet$ neurological outcome $\bullet$ prognosis • unresponsive wakefulness syndrome

\section{Causes of development of severe disorders of consciousness}

Hypoxic-ischemic encephalopathy, traumatic brain injury, intracerebral hemorrhage, subarachnoid hemorrhage and brain infarction are common causes of mortality and morbidity [1-8]. Many patients affected by these acquired brain injuries (ABIs) die in the acute stages during their stay in the intensive care unit; others, after a coma phase, usually develop a severe disorder of consciousness (DoC), characterized by an unresponsive wakefulness syndrome or a minimally conscious state (MCS). In some cases, consciousness level improves, transitioning to an emergence from MCS (E-MCS); however, patients with E-MCS often show a severe neurological disability [9]. In recent years, improvements both in intensive care technology and in neurosurgical procedures have reduced the mortality rate; however, as a result, many patients discharged from the acute setting exhibit severe DoCs.

\section{Neurological prognosis of patients with disorders of consciousness}

Predicting the neurological outcome in a patient with severe $\mathrm{DoC}$ is an important goal in the acute stages after ABI [10], mainly for two reasons: first, the appropriate therapeutic management among the intensive care units can be better identified, and second, a patient who needs further multidisciplinary rehabilitation treatment compared with a patient who should be addressed to a long-term care after hospital discharge, can be better identified [6,7].

However, establishing the neurological prognosis in postacute stages, when patients are already admitted to the intensive rehabilitation units (IRUs), is also important for individualized multidisciplinary rehabilitation planning, in view of realistic expectations of a patient's recovery and for assisting physicians in communicating with the patient's family members and caregivers. However, in contrast to the literature about the acute stages, there are no strong data about the long-term neurological prognosis in patients already admitted to the IRUs. In fact, to date, only a few studies $[9,11]$ have analyzed postacute clinical or instrumental predictors for late neurological prognosis, showing some limitations and conflicting results. One of the main reasons for the lack of postacute robust neurological predictors has been the evaluation of only one parameter at a time in most of the recent studies. In fact, only Scarpino et al. [12] accounted for the association of postacute clinical and instrumental parameters for the neurological outcome prediction, as suggested by Kotchoubey and Pavlov [13]. In particular the Coma Recovery Scale-Revised total score at patient admission in IRU and its improvement in the first 4 weeks after admission, are the only clinical variables that have been showed to be predictors of neurological outcome predictors [9]. Of the instrumental parameters, electroencephalography (EEG) has been the test most commonly investigated both for diagnostic and prognostic purposes.

Future Medicine 


\section{The prognostic value of postacute encephalography in patients with disorders of consciousness}

In contrast to neuroimaging (brain computed tomography and MRI), EEG is a simple, risk-free, and inexpensive test that can be performed at the patient's bedside. For these reasons, the EEG findings represent the neurological predictor most investigated, both in the acute and postacute phases in patients with severe ABI. The 2012 American Clinical Neurophysiology Society terminology for EEG in the critical care setting [14] was accepted by researchers as standard for identifying specific EEG patterns as indicative of poor and good prognoses in the acute stage; however, for the post-acute stage, data are still conflicting. This knowledge gap exists mainly because researchers have not agreed about specific terminology and classification of the postacute EEG findings in patients with severe DoC. Moreover, physicians have not agreed about which EEG features are most useful for prognostic purposes.

The evaluation of only one EEG descriptor at a time as a neurological predictor represents another limitation of most of the studies. Background reactivity was the EEG descriptor most investigated $[11,12,15]$ and even though tested in different ways, when observed in a standard 30 min EEG recording, it was strongly suggestive of an improvement in the long-term neurological outcome. Detectable transient patterns of stage II sleep are another EEG descriptor widely evaluated both as a diagnostic tool for the evaluation of the severity of DoC and as a late neurological predictor $[12,16,17]$. It was investigated through prolonged recordings in most of the research on this EEG feature. However, when this descriptor is investigated through a standard $30 \mathrm{~min}$ EEG recording, its presence is also related to an improvement in the neurological outcome, even with a reduced sensibility, because of the lower chance of occurrence in a short EEG recording.

Epileptic discharges have also been investigated as a neurological predictor; however, the results were found to be inconsistent. Some authors showed that when epileptic discharges were present, they hampered the recovery of consciousness [12,18] whereas Bagnato et al. [19] reported that the occurrence of structural epilepsy did not affect the recovery of consciousness.

To standardize the EEG interpretation, and therefore, to identify specific EEG descriptors as having good or poor neurological prognostic meaning, some classifications for the postacute EEG of patients with DoC have been proposed. In particular, after considering specific EEG descriptors (reactivity, voltage and frequency) as neurological predictors, Bagnato et al. [11] arbitrarily assigned a specific score to each pattern of all the descriptors, achieving a total score ranging from 3 to 7 . Higher scores were associated with the likelihood of showing an improvement in the consciousness level. Scarpino et al. [12] instead applied the 2012 American Clinical Neurophysiology Society EEG terminology [14] - which until that time had been used exclusively in the acute setting - for the interpretation of the postacute EEG. They observed that the presence of higher frequencies ( $\alpha$-waves), detectable transient patterns of stage II sleep, reactivity and variability in background EEG activity (a descriptor that had never been taken into account previously) were associated with an improvement in the consciousness level whereas the presence of epileptic discharges, lower frequencies ( $\delta$-waves) and the absence of reactivity and variability in the background activity were associated with a poor prognostic meaning. Estraneo et al. [20] also proposed a postacute EEG classification to be used only as a diagnostic tool to better assess the patient's consciousness level rather than as a prognostic tool. Their classification was based mainly on the assessment of the EEG reactivity associated with the evaluation of specific parameters of the background activity, such as frequencies, voltage or the presence of an anterior-posterior gradient. However, to date, there is no evidence as to which of these proposed classifications has the best predictive power for the long-term neurological outcome.

\section{Future perspective}

Further perspective multicenter studies are needed both to identify which classification is the most accurate in the prediction of neurological improvement and to identify which EEG descriptors have a specific prognostic meaning. The findings would help achieve standardization in interpretation of test results as already achieved in the early stages after ABI, during the coma phase. Addressing this knowledge gap could enable physicians to use the EEG as a robust postacute predictor of late neurological outcome in patients with severe DoCs who are already admitted to IRUs. Moreover, it would be worthwhile to analyze the EEGs with regard to the initial conscious state of the patients because previous data [12] has shown that some EEG descriptors had prognostic meanings only for patients with unresponsive wakefulness syndrome, but they did not show a significant prognostic meaning in patients with MCS. Moreover, other authors have reported that both the initial consciousness level as the initial CRS-R score could reflect the possibility of recovery of consciousness in the patients, regardless of the initial EEG pattern [9,12]. 


\section{Conclusion}

Prognosticating neurological outcomes is an important goal in the postacute phase when patients with severe DoC after ABI have already been admitted to the IRUs. An EEG usually performed in the first days after admission could provide both diagnostic and prognostic information. However, to date, evidence regarding the use of EEG as a postacute prognostic tool are limited and conflicting, mainly because only retrospective and monocentric studies have been performed. The EEG classifications previously proposed must be compared with to determine which is the best for postacute EEG interpretation and thus for identifying specific EEG descriptors with a specific prognostic meaning.

According to Kotchoubey and Pavlov [13], who suggested the need of a combination of clinical data and auxiliary variables for the neurological prognosis, the EEG has the potential to be the ideal instrumental test for neurological prognostication.

\section{Financial \& competing interests disclosure}

The authors have no relevant affiliations or financial involvement with any organization or entity with a financial interest in or financial conflict with the subject matter or materials discussed in the manuscript. This includes employment, consultancies, honoraria, stock ownership or options, expert testimony, grants or patents received or pending, or royalties.

No writing assistance was utilized in the production of this manuscript.

\section{Open access}

This work is licensed under the Attribution-NonCommercial-NoDerivatives 4.0 Unported License. To view a copy of this license, visit http://creativecommons.org/licenses/by-nc-nd/4.0/

\section{References}

Papers of special note have been highlighted as: $\bullet$ of interest; $\bullet \bullet$ of considerable interest

1. Carrai R, Scarpino M, Lolli F et al. Early-SEPs' amplitude reduction is reliable for poor-outcome prediction after cardiac arrest? Acta Neurol. Scand. 139, 158-165 (2019).

2. Carrai R, Grippo A, Scarpino M et al. Time-dependent and independent neurophysiological indicators of prognosis in post-anoxic coma subjects treated by therapeutic hypothermia. Minerva Anestesiol. 82, 940-949 (2016).

3. Grippo A, Carrai R, Fossi $S$ et al. Absent SEP during therapeutic hypothermia did not reappear after re-warming in comatose patients following cardiac arrest. Minerva Anestesiol. 79, 360-369 (2013).

4. Scarpino M, Lanzo G, Carrai R et al. Predictive patterns of sensory evoked potentials in comatose brain injured patients evolving to brain death. Neurophysiol. Clin. 47, 19-29 (2017).

5. Scarpino M, Grippo A, Lanzo G et al. The burden of clinical neurophysiology for the neurological prognosis of coma. Future Neurol. 13, 127-129 (2018).

- A survey of the use of EEG and Somatosensory Evoked Potentials (SEPs) for the prognostication of comatose patients in real clinical settings with practical suggestions for the increase the use of SEPs as a prognostication tool.

6. Scarpino M, Lanzo G, Lolli F et al. Data on multimodal approach for early poor outcome (Cerebral Performance Categories 3-5) prediction after cardiac arrest. Data in Brief 19, 704-711 (2018).

7. Scarpino M, Lanzo G, Lolli F et al. Neurophysiological and neuroradiological multimodal approach for early poor outcome prediction after cardiac arrest. Resuscitation 129, 114-120 (2018).

8. Scarpino M, Lolli F, Lanzo G et al. Neurophysiology and neuroimaging accurately predict poor neurological outcome within 24 hours after cardiac arrest: the ProNeCA prospective multicentre prognostication study. Resuscitation 143 115-123 (2019).

9. Portaccio E, Morrocchesi A, Romoli AM et al. Improvement on the coma recovery scale-revised during the first four weeks of hospital stay predicts outcome at discharge in intensive rehabilitation after severe brain injury. Arch. Phys. Med. Rehabil. 99, 914-919 (2018).

10. Amantini A, Carrai R, Fossi S et al. The role of early electroclinical assessment in improving the evaluation of patients with disorders of consciousness. Funct. Neurol. 26, 7-14 (2011).

- A comprehensive approach to neurophysiological prognostication of comatose patients after acquired brain Injury.

11. Bagnato S, Boccagni C, Sant'Angelo A et al. EEG predictors of outcome in patients with disorders of consciousness admitted for intensive rehabilitation. Clin. Neurophysiol. 126, 959-966 (2015).

12. Scarpino M, Lolli F, Hakiki B et al. Prognostic value of post-acute EEG in severe disorders of consciousness, using American Clinical Neurophysiology Society terminology. Neurophysiol. Clin. 49(4), 317-327 (2019).

13. Kotchoubey B, Pavlov YG. A systematic review and meta-analysis of the relationship between brain data and the outcome in disorders of consciousness. Front Neurol. 9, 315 (2018).

-. A complete survey of the use of instrumental tools for the prognostication of comatose patients. 
14. Hirsch LJ, LaRoche SM, Gaspard N et al. American Clinical Neurophysiology Society's standardized critical care EEG terminology: 2012 version. J. Clin. Neurophysiol. 30, 1-27 (2013).

-. A detailed classification of EEG patterns occurring in comatose patients. The detailed criteria of EEG description allow the use of EEG for coma prognosis.

15. Bagnato S, Boccagni C, Prestandrea C et al. Changes in standard electroencephalograms parallel consciousness improvements in patients with unresponsive wakefulness syndrome. Arch. Phys. Med. Rehabil. 98, 665-672 (2017).

16. Pavlov YG, Gais S, Müller F et al. Night sleep in patients with vegetative state. J. Sleep Res. 26, 629-640 (2017).

17. Arnaldi D, Terzaghi M, Cremascoli $\mathrm{R}$ et al. The prognostic value of sleep patterns in disorders of consciousness in the sub-acute phase. Clin. Neurophysiol. 127, 1445-1451 (2016).

18. Pascarella A, Trojano L, Loreto V et al. Long-term outcome of patients with disorders of consciousness with and without epileptiform activity and seizures: a prospective single centre cohort study. J. Neurol. 263, 2048-2056 (2016).

19. Bagnato S, Boccagni C, Galardi G. Structural epilepsy occurrence in vegetative and minimally conscious states. Epilepsy Res. 103, 106-109 (2013).

20. Estraneo A, Loreto V, Guarino I et al. Standard EEG in diagnostic process of prolonged disorders of consciousness. Clin. Neurophysiol. 127, 2379-2385 (2016). 